\title{
SUMMATION OF SEQUENCES AND SUMMATION OF SERIES
}

\author{
G. G. LORENTZ AND K. ZELLER ${ }^{1}$
}

1. Introduction. There are two ways in which infinite matrices appear in summability theory. A sequence method of summation with the matrix $A=\left(a_{n k}\right)$ is based on the transformation

$$
\sigma_{n}=A_{n} s=\sum_{k=0}^{\infty} a_{n k} s_{k}, \quad n=0,1, \cdots,
$$

of the sequence $s=\left\{s_{n}\right\}$ into the sequence $A s=\left\{\sigma_{n}\right\}$; and a series method with the matrix $A^{\prime}=\left(a_{n k}^{\prime}\right)$ corresponds to the transformation

$$
\tau_{n}=\sum_{k=0}^{\infty} a_{n k}^{\prime} u_{k}, \quad n=0,1, \cdots,
$$

of the series $\sum u_{n}, u_{n}=s_{n}-s_{n-1}, s_{-1}=0$, into the sequence $\left\{\tau_{n}\right\}$. Conditions for the regularity of the methods $A, A^{\prime}$ are well known [1, pp. 64, 68], [2, pp. 389, 396].

If the summability field $\mathfrak{A}$ of a sequence or series method $A$ (i.e., the set of all sequences $s$, summable by $A$ ) is contained in the summability field $\mathfrak{B}$ of another method $B$ (i.e., if $\mathfrak{A} \subset \mathfrak{B}$ ), we call $B$ stronger than $A$.

It is natural to call the matrix $A^{\prime}$ of (2) dual to the matrix $A$ of (1) (or $A$ dual to $A^{\prime}$ ) if $\sigma_{n}$ becomes $\tau_{n}$ (or, respectively, $\tau_{n}$ becomes $\sigma_{n}$ ) under the application of the formal summation by parts; this is equivalent to the relation $a_{n \mathbf{k}}^{\prime}=\sum_{i=\mathbf{k}}^{\infty} a_{n i}$ (or to the relation $a_{n k}$ $\left.=a_{n \mathbf{k}}^{\prime}-a_{n, \mathbf{k}+1}^{\prime}\right)$. In many concrete cases, dual methods of summation are equivalent, in the sense that they define the same summability fields and the same limits. One can also give simple sufficient conditions which guarantee this ([4, Theorems 8,9$]$, a misprint should be corrected there: $A$ is to be replaced by $B$ ). It is easy to give examples of dual methods of summation of opposite types which are not equivalent in this sense, but this does not exclude the possibility that one of them is equivalent to some other method of the opposite type.

In this paper we show that sequence and series methods are essentially different:

There exist regular sequence summation methods for which the summability field is not contained in the summability field of any regular

Received by the editors, April 20, 1963.

1 This work has been supported by the Office of Scientific Research, U. S. Air Force, through the grant no. AF-AFOSR-62-138. 
series summation method. On the other hand there are regular series methods for which the summability field is not contained in the summability field of any regular sequence summation method.

Our problem proves to be related to (and more general than) the problem discussed by Erdös and Piranian. In [3] they give a sequence method for which there is no stronger row-finite method. Our examples are based upon matrices of types similar to theirs.

Let $A=\left(a_{n k}\right)$ be an infinite matrix with real or complex entries. For each $n=0,1, \cdots, K_{n}$ will denote the set of $k, k=0,1, \cdots$, for which $a_{n k} \neq 0$. About the matrix $A$ we shall always assume that:

(a) Each set $K_{n}$ is infinite.

( $\beta$ ) For $n \neq m$, the sets $K_{n}, K_{m}$ have a finite intersection.

Sometimes we shall also assume:

( $\gamma) \sum_{k=0}^{\infty}\left|a_{n k}\right|<+\infty$ for each $n$.

( $\delta)$ For each $n$, the union $K_{0} \cup K_{1} \cup \cdots \cup K_{n}$ contains only a finite number of adjacent integers $k, k+1$.

It is easy to find examples of regular methods which satisfy all conditions $(\alpha)-(\delta)$. The matrix of Erdös and Piranian [3, p. 397] can serve for the sequence methods; with sufficiently many first elements of each row replaced by ones, one obtains from it an example for the series methods.

2. The basic lemma. The following statement applies to matrices $A$ which satisfy $(\alpha),(\beta)$ :

Lemma. Let $B$ be a row-finite matrix such that each sequence $s=\left\{s_{k}\right\}$ with the property $A s=0$ has a convergent B-transformation Bs. Then there exists a finite set $L$ and an integer $m$ such that

$$
b_{n k}=0 \text { unless } k \in L \cup K_{0} \cup \ldots \cup K_{m} .
$$

Proof. If the condition (3) is not satisfied, then for each $L$ and each $m$ there is an integer $l$ with $l \notin L \cup K_{0} \cup \ldots \cup K_{m}$ such that $b_{n l} \neq 0$ for a certain $n$.

Let $L_{n}$ denote the set of all $l$ with $b_{n l} \neq 0$. By induction, we define sequences of integers $n_{r}, l_{r}, k_{r}$ with the following properties:

$$
\begin{gathered}
n_{1}<n_{2}<\cdots ; l_{r} \in L_{n_{r}} ; \\
l_{r} \notin P_{r}=L_{0} \cup L_{1} \cup \ldots \cup L_{n_{r-1}} \cup K_{0} \cup \ldots \cup K_{r-1} ; \\
b_{n_{r} l_{r}} \neq 0 ; k_{r} \in K_{r} ; k_{r} \notin L_{n_{r}} \cup P_{r} .
\end{gathered}
$$

At the $r$ th step, we first take $l_{r} \notin P_{r}$ in such a way that $b_{n l_{r}} \neq 0$ for a certain $n$. We must have $n>n_{r-1}$, and we put $n_{r}=n$. Then we select 
$k_{r} \in K_{r}$ with the required properties; this is possible because of $(\alpha)$ and $(\beta)$.

We now define inductively the values of $s_{k}$ on the sets $L_{n_{0}}, K_{0}$, $L_{n_{1}}, K_{1}, \ldots$. At the $r$ th step, when the values of $s_{k}$ for $k \in L_{n_{0}} \cup \ldots$. $\cup L_{n_{r-1}} \cup K_{0} \cup \ldots \cup K_{r-1}$ are already known, we first extend $s_{k}$ onto $L_{n_{r}}$ so as to have $B_{n_{r}} s=(-1)^{r}$, and then onto $K_{r}$ so that $A_{r} s=0$; we can assume that $s_{k}=0$ for all but finitely many $k$ in $K_{r}$. We complete the definition of $s_{k}$ arbitrarily outside of all sets $L_{n_{r}}, K_{r}$. We will have $A s=0$, while $B_{n_{r}} s$ diverges, a contradiction to the assumption.

3. The main results. The first theorem is essentially due to Erdös and Piranian [3].

THEOREM 1. If $A$ is a sequence method which satisfies $(\alpha),(\beta),(\gamma)$, there does not exist a row-finite regular sequence method $B$ which is stronger than $A$.

Proof. Assume that $B$ is a row-finite method with $\mathfrak{B} \supset \mathfrak{A}$. Let $L, m$ correspond to $B$ according to the lemma. For an arbitrary bounded sequence $s$, there is another sequence $s^{\prime}$ (constructed below) which coincides with $s$ on $P=L \cup K_{0} \cup \ldots \cup K_{m}$ such that

$$
A_{n} s^{\prime}=0 \quad \text { for } n>m \text {. }
$$

Since $\mathfrak{B} \supset \mathfrak{A}$, the sequence $s^{\prime}$ and hence $s$ is $B$-summable. Thus $B$ cannot be regular.

To obtain $s^{\prime}$, we select for each $n>m, k_{n} \in K_{n}, k_{n} \notin L \cup K_{0} \cup \ldots$ $\cup K_{n-1}$. We put $s_{k}^{\prime}=0$ for $k \notin P, k \neq k_{n}, n \geqq m$. It is then possible to select the $s_{k n}^{\prime}$ to satisfy (5).

TheOREM 2. If a series method $A^{\prime}$ satisfies $(\alpha),(\beta)$, there does not exist a row-finite regular series method $B^{\prime}$ which is stronger than $A^{\prime}$.

Proof. According to the lemma, we must have $\lim _{n \rightarrow \infty} b_{n k}^{\prime}=0$ for infinitely many $k$, and this contradicts the regularity of $B^{\prime}$.

Theorem 3. If a sequence method $A$ satisfies $(\alpha),(\beta),(\gamma),(\delta)$, then there does not exist a regular series method $A^{\prime}$ which is stronger than $A$.

Proof. For each fixed $n$, the limit

$$
\lim _{p \rightarrow \infty} \sum_{k=0}^{p} a_{n k}^{\prime} u_{k}=\lim _{p \rightarrow \infty}\left\{\sum_{k=0}^{p-1}\left(a_{n k}^{\prime}-a_{n, k+1}^{\prime}\right) s_{k}+a_{n p}^{\prime} s_{p}\right\}
$$

must exist for all $s \in \mathfrak{A}$. It follows from the lemma that for some $m$ and some finite set $L$, 


$$
\begin{gathered}
a_{n p}^{\prime}=0, p \notin P, a_{n k}^{\prime}-a_{n, k+1}^{\prime}=0, k \notin P, \\
P=L \cup K_{0} \cup \cdots \cup K_{m} .
\end{gathered}
$$

From $(\delta)$ we see that, for each fixed $n$, only finitely many $a_{n z}^{\prime}$ are different from zero. An application of Theorem 1 completes the proof.

REMARK. Condition $(\delta)$ cannot be omitted from the assumptions of Theorem 3. In fact, one can find regular matrices $A$ which satisfy $(\alpha)$, $(\beta)$, and $[4,(29)]$; because of the last condition, each such $A$ is weaker than its dual $A^{\prime}$.

THEOREM 4. If a series method $A^{\prime}$ satisfies $(\alpha),(\beta)$, then there is no sequence method $A$ with $\mathfrak{A} \supset \mathfrak{A}^{\prime}$.

Proof. Let $n$ be fixed, then

$$
\lim _{p \rightarrow \infty} \sum_{k=0}^{p} a_{n k} s_{k}=\lim _{p \rightarrow \infty} \sum_{k=0}^{p}\left(\sum_{i=k}^{p} a_{n i}\right) u_{k}
$$

must exist for all sequences $u=\left\{u_{k}\right\}$ for which the $A^{\prime}$-transform $A^{\prime} u$ exists and is equal to zero. According to the lemma, there are $m$ and $L$ such that for $P=L \cup K_{0} \cup \ldots \cup K_{m}$,

$$
a_{n k}+a_{n, k+1}+\cdots+a_{n p}=0 \quad \text { for } k \notin P, p \geqq k \text {. }
$$

There exists a $k_{0} \notin P$, and then (9) implies $a_{n k_{0}}=a_{n, k_{0}+1}=\cdots=0$. Thus $A$ is row-finite, and our statement follows from Theorem 2.

\section{REFERENCES}

1. R. G. Cooke, Infinite matrices and sequence spaces, Macmillan, London, 1950.

2. P. Dienes, The Taylor series, Dover, New York, 1957.

3. P. Erdös and G. Piranian, Convergence fields of row-finite and row-infinite Toeplitz transformations, Proc. Amer. Math. Soc. 3 (1950), 397-401.

4. G. G. Lorentz, Über Verfahren, die von einem Stieltjes-Integral abhängen, Acta Math. 79 (1947), 255-272.

\section{SYRACUSE UNIVERSITY AND}

TübIngen University, TübIngen, Germany 